\title{
Next-generation tracking system for future hadron colliders
}

\section{Sola*, N. Cartiglia, M. Mandurrino}

INFN Sezione di Torino

\section{R. Arcidiacono, M. Ferrero}

Università del Piemonte Orientale, Novara, and INFN Sezione di Torino

\section{F. Siviero, M. Tornago}

Università degli Studi di Torino and INFN Sezione di Torino

E-mail: valentina.solaeto.infn.it

The design of future high-energy and high-intensity hadronic machines, such as FCC-hh, relies on the ability of detectors to sustain harsh radiation environments while keeping excellent performances on tracking and tagging all the interaction products. In order to face the challenge, a vast $\mathrm{R} \& \mathrm{D}$ effort is required.

In this paper, we propose a novel concept of tracking system, that combines the possibility to track particles up to fluences of the order of $10^{17} \mathrm{n}_{e q} / \mathrm{cm}^{2}$ together with a precise time information, $\sigma_{t} \sim$ 10 ps. For this purpose, Low-Gain Avalanche Diodes (LGAD) are the suited technology.

For the innermost, most irradiated portion of the detector, very thin sensors $(20-40 \mu \mathrm{m})$ with moderate gain $(\sim 5-10)$ can provide the required tolerance to the radiation. For such detectors, the internal gain mechanism of LGAD allows to provide the same amount of charge released by a particle passing $100-200 \mu \mathrm{m}$ of standard PiN diodes up to $\Phi \sim 0.5 \cdot 10^{16} \mathrm{n}_{e q} / \mathrm{cm}^{2}$. Above those fluences, the thin doped layer responsible for the signal multiplication gets deactivated, but if operated at the proper bias voltage $(\sim 500 \mathrm{~V})$ the signal multiplication happens inside the whole irradiated bulk volume.

Moreover, in the region of the tracker detector where the level of overall fluence keeps $\leq 0.5-$ $1 \cdot 10^{16} \mathrm{n}_{e q} / \mathrm{cm}^{2}$, LGAD with a geometry optimised for timing measurement, the so-called UltraFast Silicon Detectors (UFSD), can be used to provide precise position and timing information at the same time. Considering the current timing performances of UFSD under irradiation and assuming a $\sigma_{t} \sim 40$ ps from sensor + ASIC, the usage of track-timing layers alternated to tracking only layers can provide an ultimate $\sigma_{t} \sim 10$ ps per single track.

The 28th International Workshop on Vertex Detectors - Vertex2019

13-18 October, 2019

Lopud, Croatia

${ }^{*}$ Speaker. 


\section{Introduction}

Since their advent in particle physics experiments, silicon detectors have been a key tool in tracking charged particles with high precision [1]. Their development culmiated in the construction of very large scale detectors at the LHC experiments. A lot of effort has been invested over the last decade to extend the understanding of radiation damage in silicon to the maximum fluences foreseen at the High Luminosity LHC (HL-LHC), of about $\Phi \sim 2 \cdot 10^{16} \mathrm{n}_{e q} / \mathrm{cm}^{2}$.

To build HL-LHC tracking detectors the $\mathrm{n}$-in-p technology has been chosen for planar sensors as it demonstrated to be more tolerant to radiation with respect to the p-in-n in use at the LHC [2], and the 3D sensor technology [3] is considered for the innermost layer. However, three effects are currently limiting the use of silicon detectors much above $10^{16} \mathrm{n}_{e q} / \mathrm{cm}^{2}$ : (i) the loss of charge collection efficiency due to charge carrier trapping in the damaged bulk, (ii) the inhability to properly deplete the sensor bulk due to the creation by irradiation of acceptor states, and (iii) the distortion of the electric field due to the trapping of the charge carriers, leading to the so-called double junction.

Future hadron colliders, such as the hadronic Future Circular Collider (FCC-hh) [4], will require radiation tolerance of the tracking system exceeding that of present detectors by more than an order of magnitude, up to fluences of $\sim 5 \cdot 10^{17} \mathrm{n}_{e q} / \mathrm{cm}^{2}$, as shown in Fig.1. At present, there are no sensors able to efficiently work at that fluence level, and a vast R\&D effort is needed to understand the behaviour of silicon sensors under extreme irradiation and to design the next generation of silicon detectors which can operate in such harsh radiation environments.

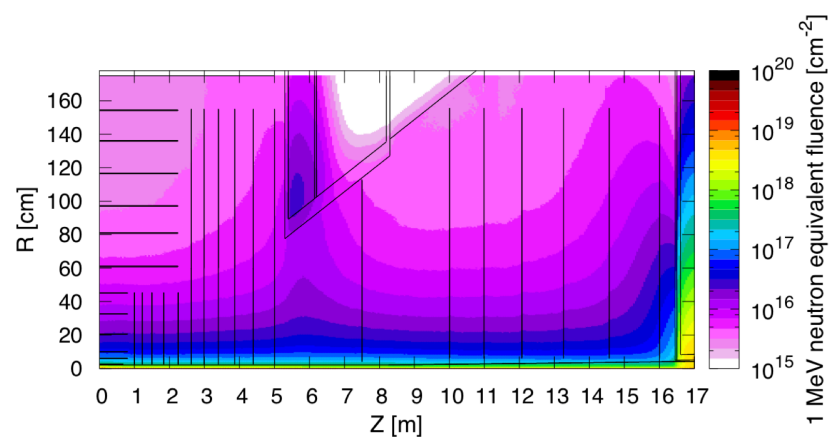

Figure 1: Radiation budget simulation for the FCC-hh tracker region after an integrated luminosity of $30 \mathrm{ab}^{-1}$ (figure by M.I. Besana).

\section{Tracking at extreme fluences}

To efficiently record a particle hit, the best performing electronics under development for the HL-LHC tracking systems requires at least $1 \mathrm{fC}$ of charge from the sensor [5]. Therefore, to build a detector able to work up to $\Phi=5 \cdot 10^{17} \mathrm{n}_{e q} / \mathrm{cm}^{2}$, it is necessary to design sensors which crossed by a particle can provide the required charge over the entire lifetime [6].

\subsection{Saturation of radiation damage}

The present studies of radiation damage on silicon are performed on thick sensors, with active thickness of $\sim 100-200 \mu \mathrm{m}$, and this fact complicates the understanding of the basic effects of 

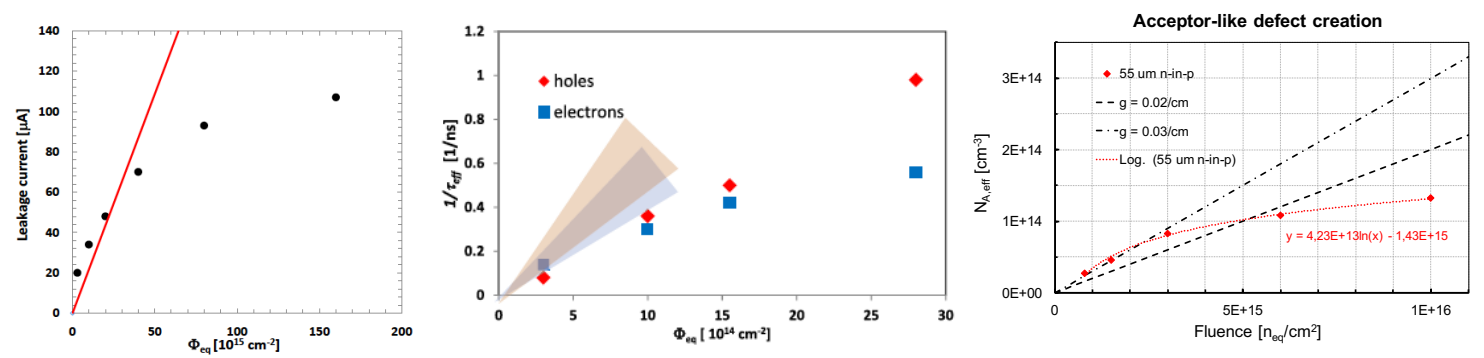

Figure 2: Evolution of the radiation damage as a function of the equivalent fluence: increase of the dark current [7] (left); increase of the charge carrier trapping probability [8] (centre); increase of the effective acceptor density [9] (right).

radiation damage, since at high fluences, $\sim 10^{16} \mathrm{n}_{e q} / \mathrm{cm}^{2}$, thick sensors cannot be depleted and the dark current distorts the electric field. Despite this shortcomings, recent studies show that at fluences above $5 \cdot 10^{15} \mathrm{n}_{e q} / \mathrm{cm}^{2}$, silicon behaves better than predicted from models built on low fluence data. In particular, Fig. 2 shows that (i) the dark current increase with fluences tends to saturate, (ii) the measured trapping probability is lower than predictions, especialy for electrons, allowing for a higher-than-expected charge collection efficiency, and (iii) the increase of effective acceptor density of the bulk substrate slows down.

This better-than-expected behaviour could be explained as a saturation of the radiation damage in the silicon lattice: considering the definiton of the silicon radius, $r_{S i}=1.18 \cdot 10^{-8} \mathrm{~cm}$, as the minimum distance between two silicon atoms, the probability that a circle with radius $r_{s i}$ around each atom has been crossed by radiaiton becomes 1 at $\Phi \sim 10^{16} \mathrm{n}_{e q} / \mathrm{cm}^{2}$, as shown in Fig.3 (left). Nevertheless, radiation damages on an already damaged lattice have to be investigated.

The evolution of the effective acceptor density with irradiation can be parametrised as follow:

$$
N_{A, e f f}(\Phi)=g_{c} \cdot \Phi+N_{A, e f f}(0) \cdot e^{-c \cdot \Phi},
$$

where $\Phi$ is the irradiation fluence, $N_{A, e f f}(0)\left(N_{A, e f f}(\Phi)\right)$ the initial (after a fluence $\Phi$ ) effective acceptor density, $g_{c}$ is the introduction rate for the space charge, and $c$ is the removal coefficient
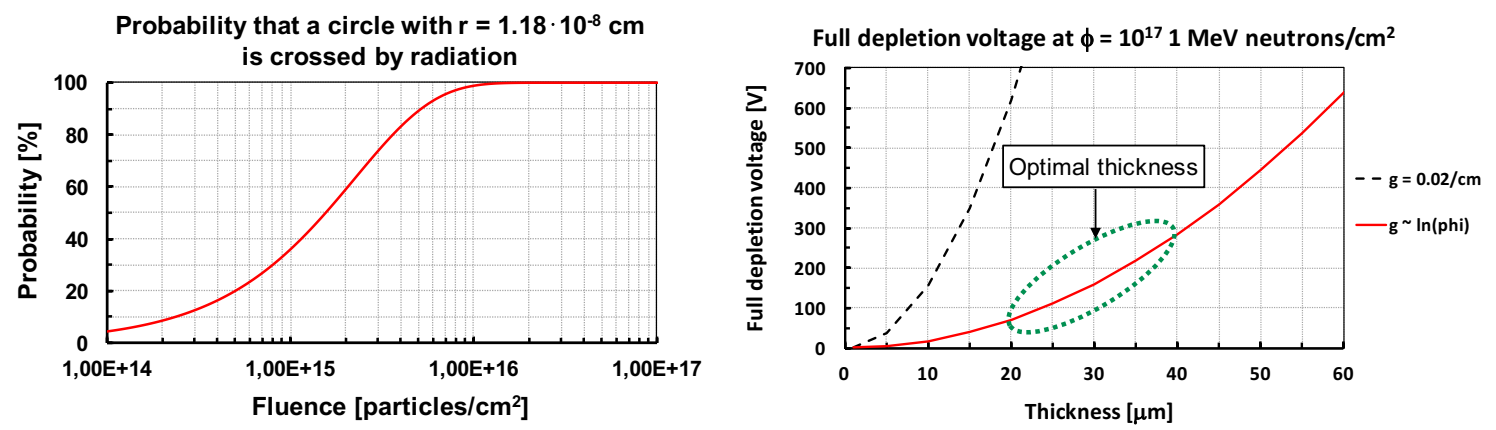

Figure 3: Left: probability that a circular surface with $\mathrm{r}=1.18 \cdot 10^{-8} \mathrm{~cm}$ is crossed by a particle, as a function of the fluence. Right: predicted $\mathrm{V}_{F D}$ as a function of sensor thickness at a fluence of $10^{17} \mathrm{n}_{e q} / \mathrm{cm}^{2}$, calculating the bulk effective acceptor density using the linear increase with $\mathrm{g}_{c}=0.02 / \mathrm{cm}$ (black dashed line), and $\mathrm{g}_{c}$ extrapolated from the data [9] (red line). 
depending on the initial acceptor density and on the radiation type [10]. For high resistivity substrates, only the first term of Eq.(2.1) contributes to the effective acceptor density evolution, and its increase directly impacts on the operation of irradiates sensors. The bias voltage needed to fully deplete the sensor volume linearly depends on the effective doping of the substrate, $N_{\text {eff }}$, and quadratically increases with the sensor thickness, $d$ :

$$
V_{F D}=\frac{e\left|N_{e f f}\right| d^{2}}{2 \varepsilon},
$$

where $e$ is the electron charge, and $\varepsilon$ is the silicon dielectric constant. From Fig.3 (right) it is possible to grasp how the radiation damage saturation directly affects the sensor operation: at a fluence of $10^{17} \mathrm{n}_{e q} / \mathrm{cm}^{2}$, with $g_{c}=0.02 / \mathrm{cm}$ as taken from litterature [8], more than $500 \mathrm{~V}$ are needed to fully deplete sensors thicker than $20 \mu \mathrm{m}$, while using a saturated $g_{c}$ as extracted from Fig.2 (right), it is possible to deplete more than $50 \mu \mathrm{m}$ while keeping the bias voltage lower than $500 \mathrm{~V}$. This saturation effects allows for collecting the charge generated in the whole sensor volume, and it make possible to increase the electric field and consequently the charge carrier drift velocity, reducing the trapping probability. Furthermore, as the dark current directly depends on the sensor volume, its impact is smaller in thin sensors.

It is worth noting that minimising the dark current contribution and the operating bias of the sensor directly reduces the power dissipation of the system: extrapolating the dark current contribution from existing data, about $1 \mathrm{~mA} / \mathrm{cm}^{2}$ is expected for a $20 \mu \mathrm{m}$ thick sensor irradiated at $10^{17}$ $\mathrm{n}_{e q} / \mathrm{cm}^{2}$ and operated at $-20^{\circ} \mathrm{C}$. If the sensors can be efficiently operated below $500 \mathrm{~V}$, the power consumption will be up to $0.5 \mathrm{~W} / \mathrm{cm}^{2}$.

\subsection{Signal multiplication in silicon sensors}

Thin sensors, with bulk thickness of $20-40 \mu \mathrm{m}$, are beneficial from the point of view of resistance to radiation. However, the charge generated from a particle crossing a thin sensor is very small, of the order of $0.2 \mathrm{fC}$ for a minimum ionising particle crossing a $20 \mu \mathrm{m}$ thick sensor, which is clearly too small to be recorded by the electronics. Therefore, thin sensors require an internal multiplication of the signal, in order to efficiently detect a particle hit.

Internal gain due to impact ionisation occurs when the electric field inside the sensor reaches the critical value of $E_{C} \sim 25 \mathrm{~V} / \mu \mathrm{m}$. Above that value an electron drifting to the read-out electrode acquires enough kinetic energy to release additional electron-hole pairs. Signal resulting from multiplication can be calculated via:

$$
N(x)=N_{0} \cdot M=N_{0} \cdot e^{\alpha[E(x)] \cdot x},
$$

where $M$ is the signal multiplication factor, $x$ is the sensor depth from the junction, $N_{0}$ is the signal before multiplication, and $\alpha$ is the electron ionisation coefficient which is a function of the electric field. The value $\alpha(E)^{-1}$ represents the mean free path needed by a charge carrier to acquire enough kinetic energy to create an additional electron-hole pair: charge carrier multiplication can happen if the carriers drift lenght before collision with the lattice is longer than $\alpha\left(E_{c}\right)^{-1} \sim 100 \mathrm{~nm}$.

As depicted in Fig. 4, there are three methods to attain an electric field high enough to induce charge multiplication: 

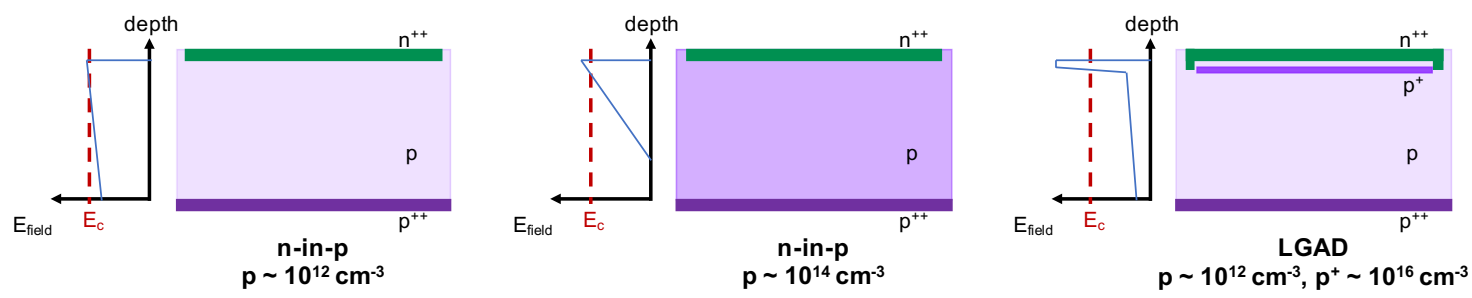

Figure 4: Sketch of n-in-p diodes with low (left) and high (centre) acceptor density in the bulk, and a LowGain Avalanche Diode (right). The electric field behaviour as a function of the sensor depth is also shown. Different tones of purple reflect the acceptor doping concentrations.

(a) For low doped bulk, the $E_{\text {field }}$ is mostly due to the applied bias. Under this circumstance, the field is almost flat, so it is either always or never close to the critical value necessary to induce impact ionisation. In this configuration, sensors tend to be very unstable, as a small variation of the bias voltage value or of the operating temperature can stop or trigger a breakdown.

(b) For highly doped bulk, the $E_{\text {field }}$ has a slope proportional to the bulk doping concentration: multiplication happens near the junction, where the field is higher. However, in this configuration the electric field increases constantly approaching the junction, therefore it can easily start an avalanche at its highest point.

(c) In the Low-Gain Avalanche Diode (LGAD) design [11], the gain layer doping produces a sudden increase of the $E_{\text {field }}$ at a distance of $1-2 \mu \mathrm{m}$ from the junction. The total field, resulting from the sum of the gain layer and the bias voltage, is near the critical value only for a short distance, it is flat and controlled by the bias value, offering a very stable configuration.

For new sensors, the main conditions to have stable gain can be summrised as follows: (i) the electric field should be near the critical value for a shor distance only, of the order of $1 \mu \mathrm{m}$, (ii) the field above the critical value should be as flat as possible, and (iii) the field value should be controlled by bias and not by bulk doping. Therefore, only the LGAD design can provide the moderate internal gain necessary for the stable operation of thin sensors.

Under irradiation, the effective acceptor density of LGAD changes accoring to Eq.(2.1): the highly doped gain layer becomes less and less doped, whereas the effective bulk doping increases
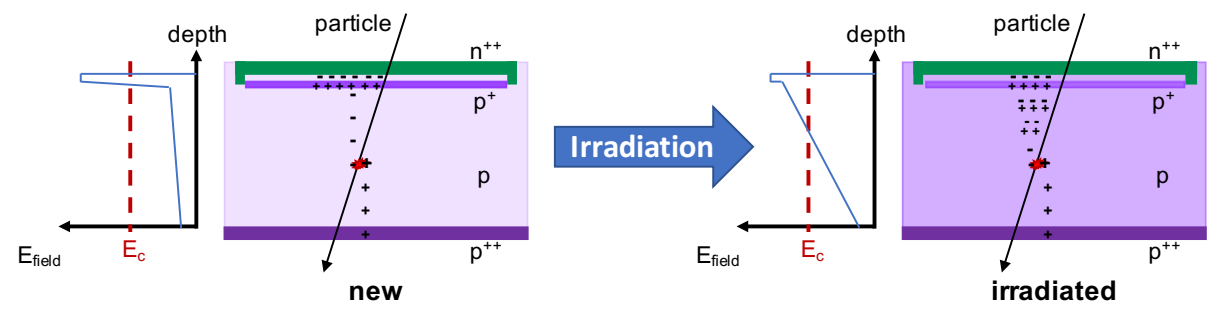

Figure 5: Sketch of a new (left) and irradiated (right) Low-Gain Avalanche Diode, together with the electric field behaviour as a function of the sensor depth. Different tones of purple reflect the p-doping concentrations in the sensor. The detail of the creation and drifting of an electron-hole pair is shown as an example. 


\section{Collected charge from irradiated LGAD - WF2}

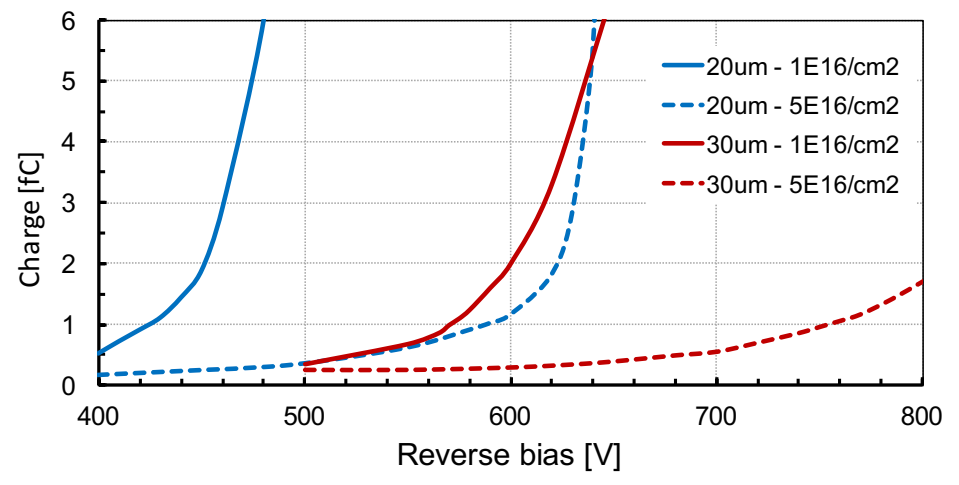

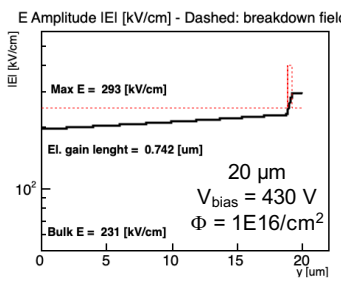

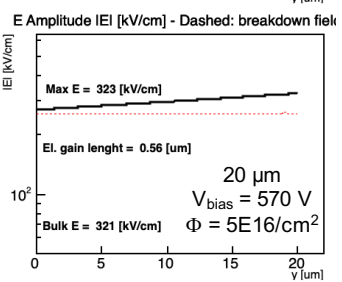

Figure 6: Left: collected charge from 20 and $30 \mu \mathrm{m}$ thick sensors irradiated at $1-5 \cdot 10^{17} \mathrm{n}_{e q} / \mathrm{cm}^{2}$ as a function of the applied bias. Right: electric field as a function of the depth for the $20 \mu \mathrm{m}$ thick sensor, at the bias voltage at which $1 \mathrm{fC}$ of charge is collected. Simulation are performed with Weightfield2 [12]; the gain layer implants have been tailored to release $5 \mathrm{fC}$ of charge at $120 \mathrm{~V}$ when the sensors are new.

(see Fig.5). As a consequence, the signal multiplication from the gain layer progressively reduces with irradiation, but at high enough fluences and bias voltage values, the gain appears from the bulk. Irradiation decreases the mean free path of charge carriers, so even at high electric fields the charge multiplication is quenched, and it is possible to obtain a moderate gain controlled by the applied bias. Multiplication in the bulk will be present up to fluences at which the mean free path of electrons and holes is longer than $\alpha(E)^{-1}$. It is crucial that the sensors can be over depleted even at the highest fluences so that the electric field remains as flat as possible when it approaches the critical value and does not lead to uncontrollable breakdown. An active thickness of $20-40 \mu \mathrm{m}$ would be ideal as it can still be depleted after a fluence of $10^{17} \mathrm{n}_{e q} / \mathrm{cm}^{2}$. At every moment in this evolution, the gain $5-10$ should be sufficient to guarantee the delivery of at least $1 \mathrm{fC}$ of charge, as shown in Fig.6 for 20 and $30 \mu \mathrm{m}$ thick sensors.

\section{Timing at extreme fluences}

LGAD with a design optimised for timing measurement, the so called Ultra-Fast Silicon Detectors (UFSD) [13], can be used to associate precise timing together with precise position information. To achieve excellent timing performances, the sensors have to provide at least $5 \mathrm{fC}$ of charge to the electronics [14]. At present, it is possible to reach a time resolution of 40 ps per single hit up to a fluence of $\sim 2 \cdot 10^{15} \mathrm{n}_{e q} / \mathrm{cm}^{2}$ [15], while the timing capability at higher fluences is limited by the gain loss due to deactivation of the gain layer as a consequence of acceptor removal (second term of Eq.(2.1)).

To maintain excellent timing performaces up to $\Phi \sim 5 \cdot 10^{16} \mathrm{n}_{e q} / \mathrm{cm}^{2}$ by using $20-40 \mu \mathrm{m}$ thick UFSD sensors it is necessary to keep an internal gain of $\sim 20$ up to the target fluence. Therefore, it is crucial to postpone the multiplication transition from the gain layer to the sensor bulk to above $10^{16} \mathrm{n}_{e q} / \mathrm{cm}^{2}$. Adding Carbon atoms in the same volume of the gain layer implant already doubled its tolerance to radiation [10], and different doses of Carbon implantation leads to different 

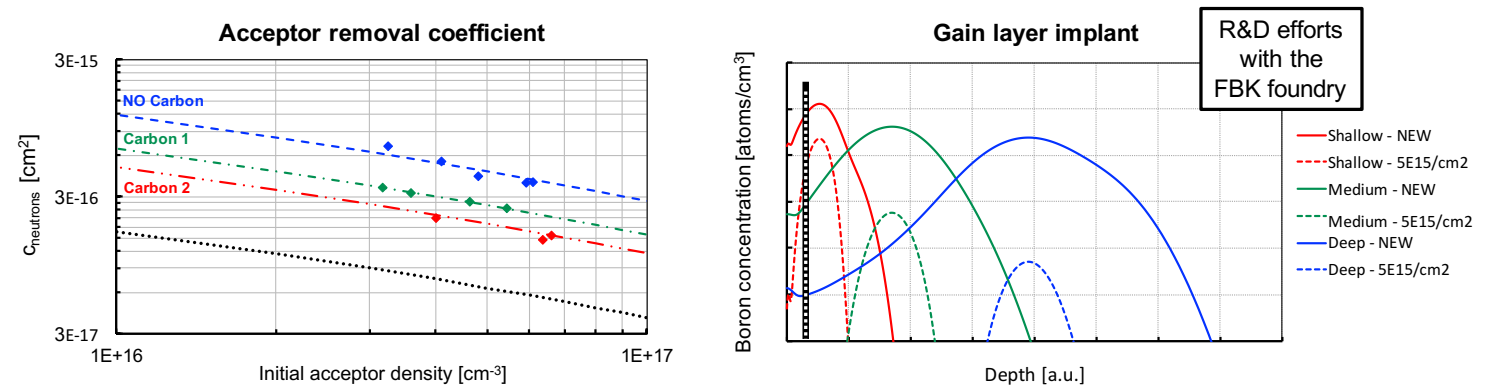

Figure 7: Left: acceptor removal coefficients, $c$, as a function of the initial acceptor density $N_{A}(0)$, for different doses of Carbon co-implanted in the gain layer volume [10]. The black dotted line shows the final target of the R\&D effort. Right: possible profiles of gain layer implanted at different depth from the sensor surface, when new and after a fluence of $5 \cdot 10^{5} \mathrm{n}_{e q} / \mathrm{cm}^{2}$. The black line represents a possible R\&D outcome: considering a conservative value of $c=2 \cdot 10^{-17} \mathrm{~cm}^{2}, 90 \%$ of the gain layer atoms will still be active after $5 \cdot 10^{5} \mathrm{n}_{e q} / \mathrm{cm}^{2}$ (white dots).

resistance to radiation, as shown in Fig.7 (left). However, in order to extend the radiation resistance of the gain layer implant of an order of magnitude in fluence, further R\&D is necessary.

Fig 7 (right) shows that different implantation depths and doping concentrations lead to different deactivation of the acceptor atoms, therefore a thinner gain layer, close to the sensor surface could bring to better resistance to radiaiton. However, the possibility of having thin gain layer implants has to cope with the Boron implantation energy and thermal budgets in the production phase.

\section{Summary}

To design the tracking detectors for the next generation of high-energy and high-intensity hadron colliders, an extensive $\mathrm{R} \& \mathrm{D}$ programme is mandatory, and the silicon sensor behaviour in the fluence regime above $10^{17} \mathrm{n}_{e q} / \mathrm{cm}^{2}$ needs to be explored and properly modelled.

The possibility to track charged particles relies on the ability to design silicon sensors which can provide at least $1 \mathrm{fC}$ of charge to the front-end electronics when crossed by minimum ionising particles, up to fluences of $\sim 5 \cdot 10^{17} \mathrm{n}_{e q} / \mathrm{cm}^{2}$. The recently observed saturation of radiation damage effects occuring above $5 \cdot 10^{15} \mathrm{n}_{e q} / \mathrm{cm}^{2}$ represents a key element for the design of such sensors. Furthermore, the design of sensors able to withstand exteme fluences relies on the possibility to use thin substrates, which are intrinsically less affected by radition, and represent the ideal environment to study the effect of radiation in the fluence regimes above $10^{16} \mathrm{n}_{e q} / \mathrm{cm}^{2}$. The usage of $20-40 \mu \mathrm{m}$ thick LGAD sensors, with internal multiplication of the signal of $\sim 5-10$, paves the way for the design of detectors which can operate in harsh radiation environments: when new, the gain layer typical of the LGAD design will be responsible for the signal muliplication; with increasing fluence the gain layer doping progressively reduces and above $5 \cdot 10^{15} \mathrm{n}_{e q} / \mathrm{cm}^{2}$ the signal multiplication occurs in the bulk region, if a sufficient bias is provided $(\sim 500 \mathrm{~V})$.

To include precise timing information of tens of picoseconds together with the tracking measurement up to extreme fluences, higher charges are required by the electronics, of the order of $5 \mathrm{fC}$. This means that the contribution of the gain layer to the signal multiplication needs to be 
extended by one order of magnitude in fluence, with respect to the tracking only case. To reach the target an extensive R\&D on the gain layer design, including an optimisation of the implantation depth and width, needs to be pursued.

\section{Aknowledgments}

Part of this work has been financed by the European Union's Horizon 2020 Research and Innovation funding program, under Grant Agreement no. 654168 (AIDA-2020) and Grant Agreement no. 669529 (ERC UFSD669529), and by the Italian Ministero degli Affari Esteri and INFN Gruppo V. The work was supported by MIUR, Dipartimenti di Eccellenza (ex L. 232/2016, art. 1, cc. 314, 337).

This work was partially performed within the CERN RD50 Collaboration, and in collaboration with the Fondazione Bruno Kessler, Trento.

\section{References}

[1] J. Kemmer, Fabrication of low noise silicon radiation detectors by the planar process, NIM A 169 (1980) 499-502 [doi : 10.1016/0029-554x(80) 90948-9].

[2] S. Terzo et al., Heavily irradiated n-in-p thin planar pixel sensors with and without active edges, JINST 9 (2014) C05023 [doi : 10 . 1088/1748-0221/9/05/C05023].

[3] J. Lange et al., Radiation hardness of small-pitch $3 D$ pixel sensors up to a fluence of $3 \times 10^{16} \mathrm{n}_{e q} / \mathrm{cm}^{2}$, JINST 13 (2018) P09009 [doi : $10.1088 / 1748-0221 / 13 / 09 /$ P 09009 ].

[4] A. Abada et al., FCC-hh: The Hadron Collider, Future Circular Collider Conceptual Design Report Vol.3, EPJ Spec.Top. 228 (2019) 755-1107 [doi: $10.1140 /$ epjst/e2019-900087-0].

[5] RD53 Collaboration, RD Collaboration Proposal: Development of pixel readout integrated circuits for extreme rate and radiation, CERN-LHCC-2013-008, LHCC-P-006 (2019).

[6] N. Cartiglia, H.F.-W. Sadrozinski, and A. Seiden, Tracking particles at fluences $5-10 \cdot 10^{16} n_{e q} / \mathrm{cm}^{2}$, PoS VERTEX2018 (2019) 029 [doi : $10.22323 / 1.348 .0029$ ].

[7] G. Kramberger et al., Charge collection studies on custom silicon detectors irradiated up to $1.6 \times 10^{16}$ $n_{e q} / \mathrm{cm}^{2}$, JINST 8 (2013) P08004 [doi: $10.1088 / 1748-0221 / 8 / 08 / \mathrm{P} 08004$ ].

[8] G. Kramberger, Reasons for high charge collection efficiency of silicon detectors at HL-LHC fluences, NIM A 924 (2018) 192-197 [doi: $10.1016 / j$.nima. 2018 .08.034].

[9] M. Ferrero et al., Recent studies and characterization on UFSD sensors, $34^{\text {th }}$ RD50 Workshop, Lancaster, UK (2019).

[10] M. Ferrero et al., Radiation resistant LGAD design, NIM A 919 (2019) 16-26 [doi:10.1016/j.nima.2018.11.121].

[11] G. Pellegrini et al., Technology developments and first measurements of Low Gain Avalanche Detectors (LGAD) for high energy physics applications, NIM A 765 (2014) 12-16 [doi:10.1016/j.nima.2014.06.008].

[12] F. Cenna et al., Weightfield2: A fast simulator for silicon and diamond solid state detector, NIM A 796 (2015) 149-153 [doi:10.1016/j.nima.2015.04.015]. 
[13] H.F.-W. Sadrozinski, A. Seiden, and N. Cartiglia, 4-Dimensional tracking with Ultra-Fast Silicon Detectors, Rep.Prog.Phys. 81 (2018) 026101 [doi : 10 . 1088/1361-6633/aa94d3].

[14] C. de la Taille et al., ALTIROCO, a 20 pico-second time resolution ASIC for the ATLAS High Granularity Timing Detector (HGTD), PoS TWEPP-17 (2018) 006 [doi: $10.22323 / 1.313 .0006]$.

[15] Z. Galloway et al., Properties of HPK UFSD after neutron irradiation up to $6 E 15 \mathrm{n} / \mathrm{cm}^{2}$, NIM A 940 (2019) 19-29 [doi:10.1016/j.nima.2019.05.017]. 